\title{
Simulation Modeling of the Tilting Mechanism of the Main Trolley of the Turnaround Charging Crane
}

\author{
V. A. Spiridonov ${ }^{1,}{ }^{*}, A . M$. Tkachenko ${ }^{1}, R . A$. Slavnitskiy ${ }^{1}$, and $A . S$. Biushkina ${ }^{1}$ \\ ${ }^{1}$ Ural Federal University named after the first President of Russia B. N. Yeltsin, Department of \\ Metallurgical and Rotary Machines, Yekaterinburg, Sverdlovsk Oblast, Russia
}

\begin{abstract}
. this article contains kinematic and dynamic analysis of the tilting mechanism of the turnaround charging crane main trolley, conducted with the use of the Mathcad package. The mechanism is modeled by a flat link mechanism - a four-bar linkage. In the same time, the analysis of the mechanism is also conducted using the Matlab Simulink environment and the tools of Multibody, which is a Simulink library. These two models were then compared. In the study the work-energy theorem and Holonomic constraints equations were used.
\end{abstract}

\section{Introduction}

Four link mechanisms are fundamental mechanisms for some types of machines. The most widespread one among the flat four-bar link mechanisms is a four-bar linkage. Each of its link connections has only one degree of freedom - rotational.

These mechanisms can be used to meet the needs of tourism or military [1], textile [2], metallurgical industries [3]. The last one is a more technically complex example than the others. As a general rule, the use of four-bar linkages is aimed at converting the rotational motion of the crank into a rocker arm tilting. This approach is most widely used in the designing of the tilting mechanism of the turnaround charging crane main trolley.

The technical requirements of an operation determine how the operating link is moved and what type of transmission mechanism, with the help of which the specific kind of movement is provided, is used. Kinematic parameters of a driven link could be changed through variation of the structure and size of the transmission mechanism links or variations in the size of a crank.

Nowadays the lack of mechanism simulation dynamic models is an urgent problem for many industrial and educational institutions. There are visual models of mechanisms made for educational purposes $[4,5]$, but the software used for the construction does not allow to determine kinematic parameters and serves only as a visual example for students. There are also models built using powerful computing and software systems - MathCAD [6] and MatLab [7, 8]; however, these examples have one common disadvantage, that is, that the models do not represent any real existing mechanisms, for which the detailed analysis is necessary in practice.

* Corresponding author: v.a.spiridonov@urfu.ru 
In spite of the deep elaboration of these works, they do not allow to analyze the dynamic characteristics taking into account the design features.

\section{Analysis of the motion of the mechanism using the analytical expressions}

In addition to building a simulation model of the mechanism in MatLab Simulink, it is possible to examine and study the behavior of the mechanism with the analytical method, using the basic laws and principles of theoretical mechanics. Analytical record of the equation of motion of the mechanism provides an opportunity to solve various tasks of the given mechanism regarding the law of motion of the driving link.

To present further calculations, Figure 1 shows the kinematic diagram of the mechanism under study, in which the link OA is a crank; the link AB is connecting rod; the link BD is a rocker arm at the end of which a bucket with the maximum permissible weight according to the datasheet is fixed; the link OS is a fixed link.

The crank OA makes a rotational motion and a complete rotation around the $\mathrm{O}$ axis. The connecting rod $\mathrm{AB}$ makes a plane-parallel motion, and the rocker arm $\mathrm{BD}$ makes a rocking motion relative to the fixed support $\mathrm{C}$.

The sizes of the links: $a=2275 \mathrm{~mm}, b=2400 \mathrm{~mm}, O A=290 \mathrm{~mm}, A B=2280 \mathrm{~mm}$; $B C=2195 \mathrm{~mm}, C D=5100 \mathrm{~mm}$.

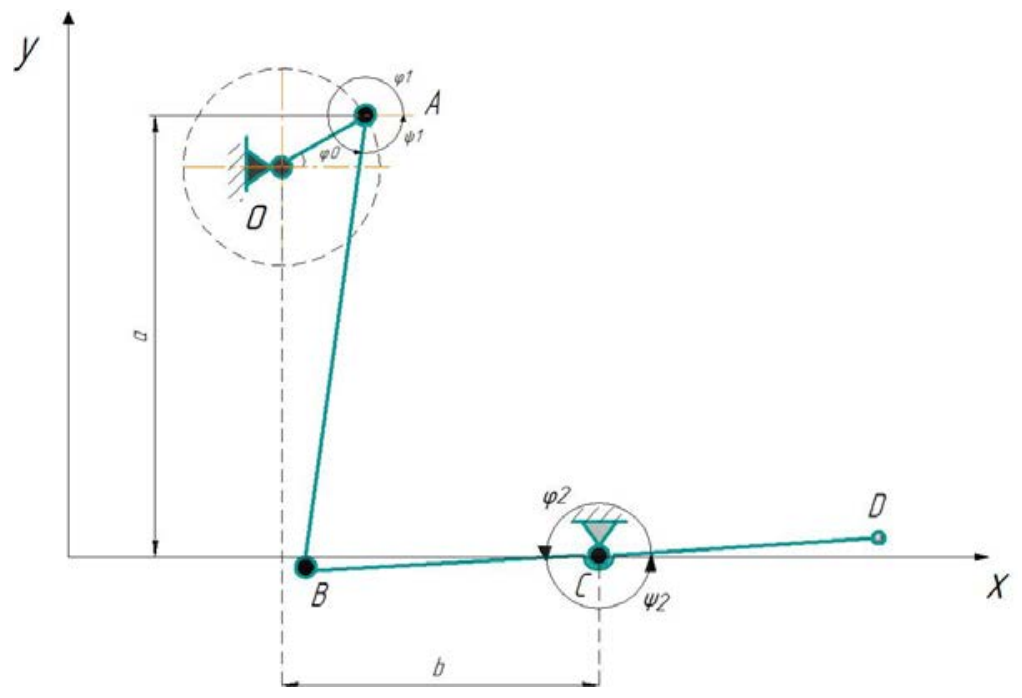

Fig. 1. The kinematic diagram of the tilting mechanism.

The driving link (crank OA) rotates with an angular velocity $\omega_{0}$, and the position of the link is characterized by an angle $\varphi_{0}$ at any time.

If the angular velocity of reduction gear shaft is constant, the angles of rotation of the links, counted from the positive $\mathrm{x}$-asis, are connected with the angles $\psi_{i}$ by the relations:

$$
\varphi_{1}=2 \pi+\psi_{1} ; \varphi_{2}=2 \pi+\psi_{2}
$$

The kinematic characteristics of the links of the mechanism in relation to the driving link can be expressed through the Holonomic constraints equations.

$\mathrm{X}$-axis:

$$
O A \cdot \cos \left(\varphi_{0}\right)+A B \cdot \cos \left(\varphi_{1}\right)=b+C B \cdot \cos \left(\varphi_{2}\right)
$$


Y-axis:

$$
O A \cdot \sin \left(\varphi_{0}\right)+A B \cdot \sin \left(\varphi_{1}\right)=a+C B \cdot \sin \left(\varphi_{2}\right)
$$

Then the angular coordinates of the links of the mechanism, expressed in terms of kinematic parameters using Holonomic constraints, have the following form:

$$
\begin{aligned}
& \varphi_{1}\left(\varphi_{0}\right)=\alpha\left(\varphi_{0}\right)+\arccos \left(\frac{C B^{2}-A B^{2}-A C\left(\varphi_{0}\right)^{2}}{2 \cdot A C\left(\varphi_{0}\right) \cdot A B}\right) \\
& \varphi_{2}\left(\varphi_{0}\right)=\alpha\left(\varphi_{0}\right)+\arccos \left(\frac{A C\left(\varphi_{0}\right)^{2}+C B^{2}-A B^{2}}{2 \cdot A C\left(\varphi_{0}\right) \cdot C B}\right)
\end{aligned}
$$

where

$$
\begin{gathered}
A C\left(\varphi_{0}\right)=\sqrt{a^{2}+b^{2}+O A^{2}-2 \cdot O A \cdot \sqrt{a^{2}+b^{2}} \cdot \cos \left(\varphi_{0}-\beta\right)} \\
\beta=\operatorname{arctg}\left(\frac{b}{a}\right) ; \\
\cos (\alpha)=\frac{O A \cdot \cos \left(\varphi_{0}\right)-b}{A C} ; \sin (\alpha)=\frac{O A \cdot \sin \left(\varphi_{0}\right)-a}{A C}
\end{gathered}
$$

The vector of unknown angular velocities, expressed through the driven link:

$$
\begin{gathered}
d \varphi_{0}\left(\varphi_{0}\right)=A\left(\varphi_{0}\right)^{-1} \cdot B\left(\varphi_{0}\right) \\
A\left(\varphi_{0}\right)=\left(\begin{array}{cc}
-A B \cdot \sin \left(\varphi_{1}\left(\varphi_{0}\right)\right) & C B \cdot \sin \left(\varphi_{2}\left(\varphi_{0}\right)\right) \\
A B \cdot \cos \left(\varphi_{1}\left(\varphi_{0}\right)\right) & -C B \cdot \cos \left(\left(\varphi_{2}\left(\varphi_{0}\right)\right)\right.
\end{array}\right) \\
B\left(\varphi_{0}\right)=\left(\begin{array}{c}
O A \cdot \sin \varphi_{0} \\
O A \cdot \cos \varphi_{0}
\end{array}\right)
\end{gathered}
$$

Then the vector of the angular velocities of the links relative to the angular velocity of the driving link is:

$$
\begin{gathered}
d \varphi_{0}\left(\varphi_{0}\right)=\left(\begin{array}{l}
\Omega_{0} \\
\Omega_{1} \\
\Omega_{2}
\end{array}\right) \\
\Omega_{i} \cdot \omega_{0}=\frac{d \varphi_{i}}{d \varphi_{0}} \dot{\varphi_{0}}=\omega_{i}
\end{gathered}
$$

Knowing the angular positions of the links at any time, as well as the angular velocities and accelerations, we can make a differential equation of the motion of the mechanism using the work-energy theorem. As this mechanism of the main trolley of the turnaround charging crane is represented with a four-bar linkage, which in its turn refers to a mechanism with one degree of freedom, we write down the work-energy theorem in the differential form.

$$
\frac{d T}{d t}=\sum_{i} N_{i}\left(\overline{F_{l}^{e}}\right)+\sum_{i} N_{i}\left(\overline{F_{l}^{\jmath}}\right)
$$

We find the kinetic energy of the entire mechanism as the sum of the kinetic energies of each link, given that the crank makes a rotational motion; the connecting rod makes a planeparallel one, which can be resolved into 2 types of motion: rotational and translational ones; the rocker arm makes a rotational motion. 


$$
\mathrm{T}=\frac{1}{2} \cdot I_{O Z} \cdot \omega_{0}{ }^{2}+\left(\frac{1}{2} \cdot I_{C 1 z} \cdot \omega_{1}{ }^{2}+\frac{1}{2} \cdot m_{1} \cdot v_{C 1}{ }^{2}\right)+\frac{1}{2} \cdot I_{C 3 z} \cdot \omega_{0}{ }^{2}
$$

The moments of inertia of the links are taken from the model made in MatLab Simulink. In general, the kinetic system will look as follows:

$$
\begin{gathered}
\mathrm{T}=\frac{1}{2} \cdot I_{\text {пр }}\left(\varphi_{0}\right) \cdot{\omega_{0}}^{2} \\
I_{\text {пр }}\left(\varphi_{0}\right)=I_{O z}+I_{C 1 z} \cdot{\Omega_{1}}^{2}+m_{1} \cdot V_{C 1}{ }^{2}+I_{C 3 z} \cdot{\Omega_{2}}^{2} ; V_{C 1}=\frac{v_{C 1}}{\omega_{0}}
\end{gathered}
$$

To solve this task, we assume that the links under consideration are absolutely solid bodies connected by perfect hinges. In this case the total power of internal forces equals zero.

$$
\sum_{i} N_{i}\left(\overline{F_{l}^{j}}\right)=0
$$

The total power of external forces equals:

$$
\sum_{i} N_{i}\left(\overline{F_{l}^{e}}\right)=N_{\text {Д }}+N_{\mathrm{H}}+\sum_{i} N_{\bar{G} i}
$$

where $N_{\text {Д }}=M_{\text {д }} \cdot \omega_{0}$ is the power transmitted from the reduction gear shaft;

$N_{\mathrm{H}}=F_{\mathrm{H}} \cdot v_{c 2}$ is the power transmitted from the load to the crane bucket;

$N_{\bar{G} i}=G_{i} \cdot v_{c i}$ is the power of gravity force of the link.

The engine torque is not constant and it is expressed in the following form:

$$
M_{\text {д }}=M_{0}-\beta \cdot \omega_{0}
$$

Then the power of external forces takes this form:

$$
\begin{gathered}
\sum_{i} N_{i}\left(\overline{F_{l}^{e}}\right)=M_{\text {пр }}\left(\varphi_{0}, \omega_{0}\right) \cdot \omega_{0}=\left(M_{\text {пр }}^{0}\left(\varphi_{0}\right)-\beta \cdot \omega_{0}\right) \cdot \omega_{0} \\
M_{\text {пр }}^{0}\left(\varphi_{0}\right)=M_{0}+F_{\mathrm{H}} \cdot V_{c 2}+\sum_{i} G_{i} \cdot V_{c i}
\end{gathered}
$$

Consequently, the equality that forms the equation of motion of the mechanism is valid:

$$
I_{\text {пр }}\left(\varphi_{0}\right) \cdot \ddot{\varphi}_{0}+\frac{1}{2} \cdot I_{\text {пр }}{ }^{\prime}\left(\varphi_{0}\right) \cdot{\dot{\varphi_{0}}}^{2}=M_{\text {пр }}\left(\varphi_{0}\right)
$$

After integrating the differential equation of motion of the mechanism, we can write the law of motion of the driving link, its angular velocity and acceleration. The graph of change in angular velocity $\omega=\omega(t)$ and in the value of the average angular velocity $\omega_{\text {cp }}$ over time is shown in Figure 2. 




Fig. 2. The graph of the changes in the angular and average velocity of the driving link over time.

\section{The building of the simulation model}

We used the MatLab Simulink software to build the dynamic computation graphs. The construction of complex three-dimensional models in the Simulink environment is a long and time-consuming process, although it has a number of advantages. In our case, there is no need to parameterize the machinery directly in MatLab, since the bench model is based on an existing mechanism, so we model the mechanism and its details in KOMPAS-3D for its subsequent integration into the dynamic environment.

It is important to note that for further convenience of working with the detailing, the world coordinates should be placed at the hinge position point of the link being modeled. When converting parts to STEP format and adding them to the File Solid block in the MatLab Simulink environment, the main coordinate system of the KOMPAS file remains also the coordinate system of the solid-state block.

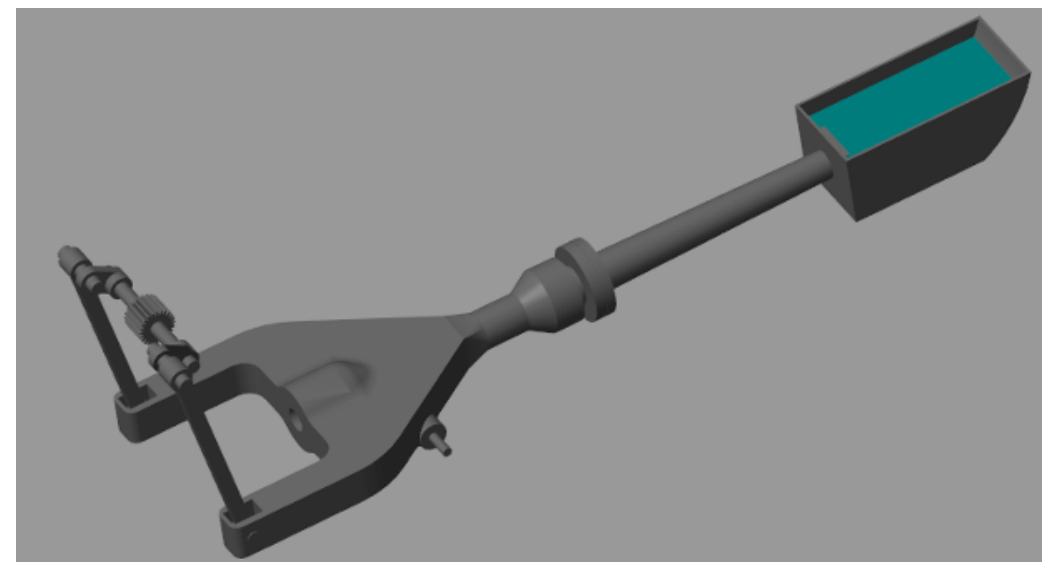

Fig. 3. Isometric projection of the tilting mechanism of the main trolley.

With the help of flowcharts we built the dynamic model, setting the engine torque, according to the formula (20).

After the done actions, it is possible to remove the necessary parameters from the built model in the nominal operating conditions (fig.3). In this case we removed the angular velocity and the torque of the crank, the angular velocity of the rocker arm and the linear velocity of the right end of the rocker arm along the vertical axis (axis Z) with the help of Scope and Transform Sensing blocks. The flowcharts are shown in Figures 4 and 5. The graphs of the removed parameters are shown in Figure 6. 


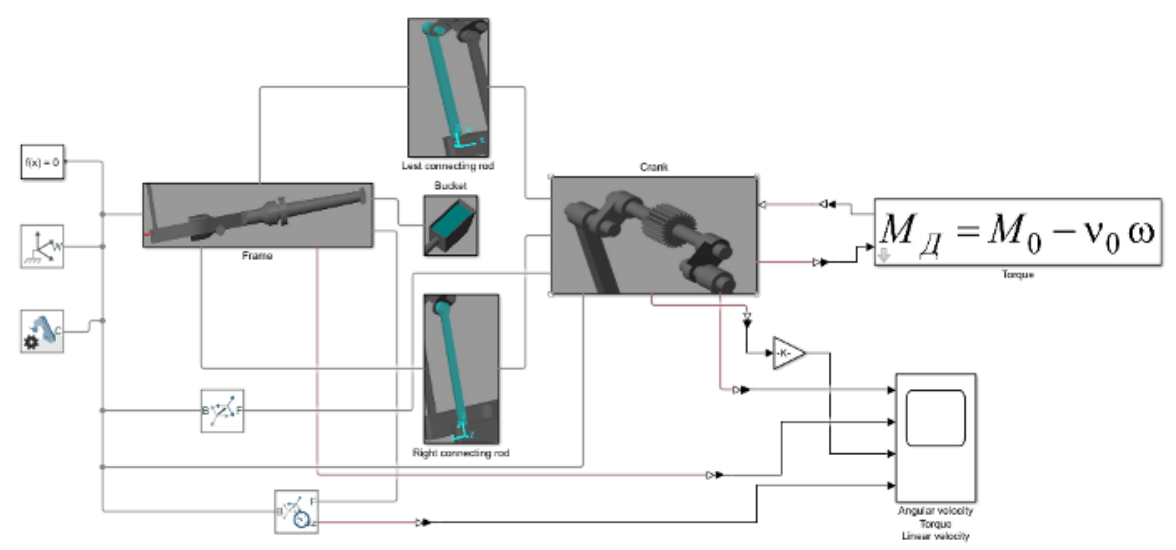

Fig. 4. Flowchart of the mechanism with the subsystems.

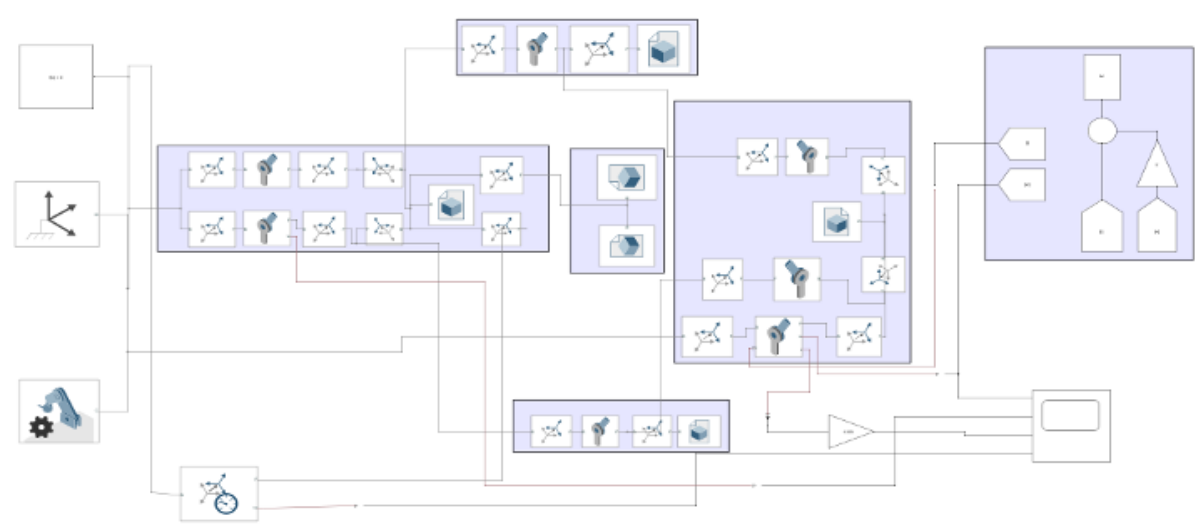

Fig. 5. Expanded flowchart of the mechanism.
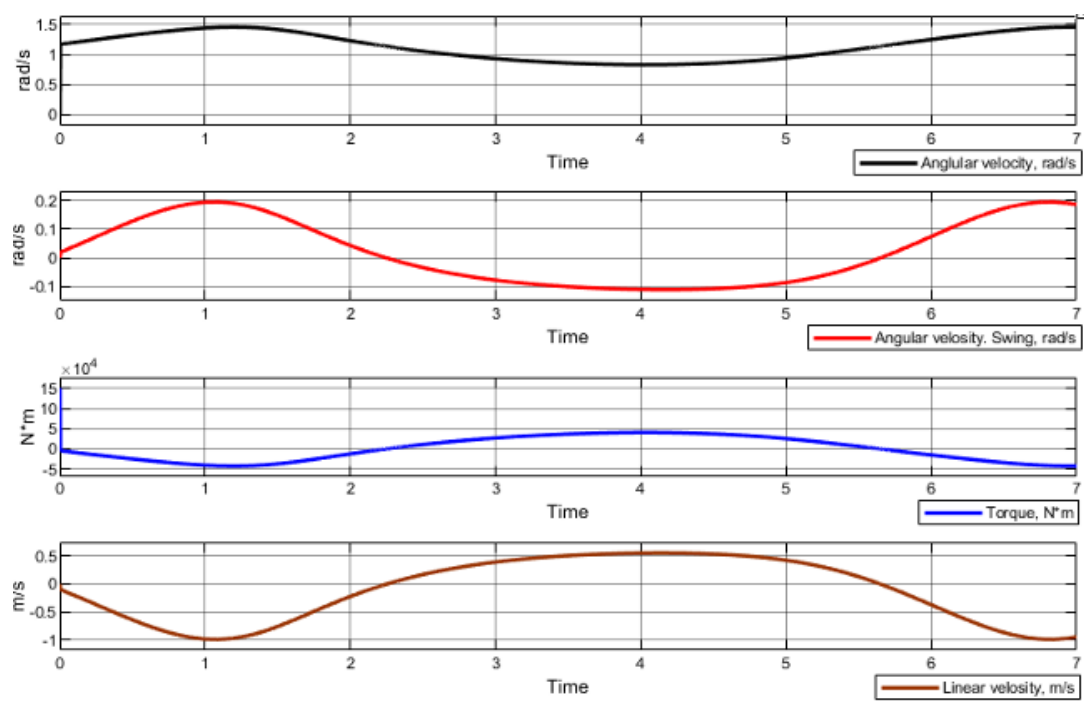

Fig. 6. Graphs of angular velocity and torque on the crank, of the linear and angular velocity of the rocker arm. 


\section{Conclusion}

Having obtained a description of the mathematical model of the turnaround charging crane tilting mechanism and having implemented numerical solutions in MathCad, as well as having built a solid-state dynamic model in MatLab, we can conclude that the two models coincide with each other.

When selecting the engine and reduction gear and when providing the starting torque for such a massive mechanism, the angular velocity of the crank, on which the velocities of all other links depend, is of the greatest importance.

The model of the tilting mechanism of the main trolley of the turnaround crarging crane, created in MatLab, can be used in the educational process, as well as to study the existing tilting mechanisms and to design the new ones.

Comparing the graph in Figure 2 and the first graph in Figure 6, we can conclude that the obtained results coincide, taking into account the errors resulting from the impossibility of checking the methods for calculating the parameters of the model in MatLab.

\section{References}

1. C. Hoberman, M. Davis. Synchronized four-bar linkages (2006)

2. V.A. Makarov, E.N, Hozina, A.N. Gavrilov. Use of four-link mechanisms as elements of lever-rod transmission of loom shed mechanisms, Textile technology №3(339), pp. 102-108

3. L.T. Dvornikov, O.S, Chuzhikov, S.P. Starikov. Crank-slider mechanism for the Press (2003)

4. V.V. Dubinin, A.V. Pashkov. Experience of creation and use of virtual models of mechanisms in the course of Theoretical Mechanics, Science and Innovation, №12(24) (2013)

5. F.N. Vulfert, B.E. Kochegarov. Computer modeling animation of mechanical system, FEFU: School of Engineering Bulletin, №1(26), pp. 24-32, (2016)

6. A. Shala, R. Likaj, M. Kelaj, X. Bajrami. Analysis of kinematics and kinetostatics of four-bar linkage mechanism based on given program, Machines. technologies. Materials, v. 6, pp. 403-408 (2017)

7. E. Vergara Hernández, R.E. Ortega Atayde, B.C. Pérez Millán, C.E. Cea Montufar. Position study of a four-bar mechanism using MATLAB, The International Journal of Engineering and Science, v. 7, pp. 63-68, (2018)

8. A.Yu. Ahpashev, V.P. Pavlov. Modeling of the link mechanisms of the excavators in the Matlab Simulink environment, IX All-Russian Conference "Youth and science" (2013) 